\title{
Integrability and the Properties of Solutions to Euler and Navier-Stokes Equations
}

\author{
L. I. Petrova \\ Moscow State University, Russia \\ E-mail: ptr@cs.msu.su
}

Received: March 14, 2012 Accepted: March 28, 2012 Online Published: May 28, 2012

doi:10.5539/jmr.v4n3p19 URL: http://dx.doi.org/10.5539/jmr.v4n3p19

\begin{abstract}
It is known that the Euler and Navier-Stokes equations, which describe flows of ideal and viscid gases, are the set of equations that are modelling the conservation laws for energy, linear momentum and mass. As it will be shown, the integrability and properties of the solutions to the Euler and Navier-Stokes equations depend, firstly, on the consistency of equations in the set of Euler and Navier-Stokes equations and, secondly, on the properties of conservation laws.

It was found that the Euler and Navier-Stokes equations have solutions of two types, namely, the solutions that are not functions (depend not only on coordinates) and generalized solutions that are functions but realized discretely, and hence, functions or their derivatives have discontinuities. A transition from the solutions of first type to generalized solutions describes the process of transition of gas-dynamic system from non-equilibrium state to the locally-equilibrium one. Such a process is accompanied by the emergence of any observable formations (such as waves, vortices, turbulent pulsations and so on), which are described by generalized solutions. This discloses the mechanism of such processes as emergence vorticity and turbulence.

These results were obtained using the relation that is deduced from the Euler and Navier-Stokes equations and contains the entropy, which specifies the state of gas-dynamic system.
\end{abstract}

Keywords: Conservation laws, Consistency of equations, Degenerate transformation, Discrete solutions

\section{Analysis of the Consistency of Equations in the Set of Euler and Navier-Stokes Equations. Evolutionary Relation}

Integrability and the properties of solutions to the set of differential equations depend on whether or not the equations in the set of differential equations are consistent. (It should be noted that the functional properties of solutions to differential equations in addition depend on whether or not the derivatives of differential equations are conjugated.)

Let us consider the gas-dynamic systems for ideal (inviscid) and viscous gases that are respectively described by Euler or Navier-Stokes equations, which simulate the conservation laws for energy, linear momentum and mass.

Assume that the gas is a thermodynamic system in the state of local equilibrium, that is, the following relation is fulfilled Haywood, R. W. (1980):

$$
T d s=d e+p d V
$$

where $T, p$ and $V$ are the temperature, the pressure and the gas volume, $s$ and $e$ are entropy and internal energy per unit volume.

Here it should be emphasized that this relation describes the state of gas particles only. That is, it is assumed that the gas particles are in the equilibrium state, whereas the gas-dynamic system itself may be in the non-equilibrium state.

The problem of consistency of equations can be studied only with the help of two nonequivalent frames of reference.

Let us introduce two frames of reference: an inertial one, which is not connected with the gas-dynamic system, and an accompanying frame of reference being connected with the manifold made up by the trajectories of the system elements. (Both Euler's and Lagrange's systems of coordinates can be examples of such frames.) 
Let us analyze the consistency of the equations that describe the conservation laws for energy and linear momentum.

In the inertial frame of reference the equation of conservation law for energy can be written as

$$
\frac{D h}{D t}-\frac{1}{\rho} \frac{D p}{D t}=A_{1}
$$

where $D / D t$ is the total derivative with respect to time, $\rho=1 / V$ and $h$ are respectively the density and enthalpy of the gas.

In the case of ideal gas described by the Euler equations we have

$$
A_{1}=0
$$

And in the case of viscous heat-conducting gas described the Navier-Stokes equations expression $A_{1}$ can be written as (see Clark, J. F. \& Machesney, M., 1964, Chapter 6, formulae (6.2.4))

$$
A_{1}=\frac{1}{\rho} \frac{\partial}{\partial x_{i}}\left(-\frac{q_{i}}{T}\right)-\frac{q_{i}}{\rho T} \frac{\partial T}{\partial x_{i}}+\frac{\tau_{k i}}{\rho} \frac{\partial u_{i}}{\partial x_{k}}
$$

Here $q_{i}$ is the heat flux and $\tau_{k i}$ is the viscous stress tensor.

Expressing enthalpy in terms of internal energy $e$ with the help of formula $h=e+p / \rho$ and using relation (1), equation (2) of the conservation law for energy can be redused to the form

$$
\frac{D s}{D t}=A_{1}
$$

Since the total derivative with respect to time is that along the trajectory, in the accompanying frame of reference the equation of the conservation law for energy takes the form:

$$
\frac{\partial s}{\partial \xi^{1}}=A_{1}
$$

where $\xi^{1}$ is the coordinate along the trajectory.

In the accompanying frame of reference the equation of conservation law for linear momentum can be presented as

$$
\frac{\partial s}{\partial \xi^{v}}=A_{v}
$$

where $\xi^{v}$ is the coordinate in the direction normal to the trajectory.

In the case of two-dimensional ideal gas, from the Euler and Navier-Stokes equations one can obtain the following expression for coefficient $A_{v}$ (see Clark, J. F. \& Machesney, M., 1964, Chapter 6, formulae (6.7.12)):

$$
A_{v}=\frac{\partial h_{0}}{\partial \xi^{v}}+\left(u_{1}^{2}+u_{2}^{2}\right)^{1 / 2} \zeta-F_{v}+\frac{\partial U_{v}}{\partial t}
$$

where $\zeta=\partial u_{2} / \partial x-\partial u_{1} / \partial y$.

In more general case the expression for $A_{v}$ can be obtained using the Crocco formulae Liepman, H. W. \& Roshko, A. (1957)

$$
\operatorname{grad} s=\left(\operatorname{grad} h_{0}+\mathbf{U} \times \operatorname{rot} \mathbf{U}-\mathbf{F}+\partial \mathbf{U} / \partial t\right) / T
$$

where $\mathbf{U}$ is the velocity of gas particle, $h_{0}=(\mathbf{U} \cdot \mathbf{U}) / 2+h, \mathbf{F}$ is the mass force. To the expression $A_{v}$ it is assigned the right-hand part of equation (9). (Note that such form of writing $A_{v}$ is enough for analysis of consistency of the equations describing the conservation laws for energy and linear momentum.)

In the case of viscous gas, the expression $A_{v}$ includes additional terms related to viscosity and heat-conductivity.

From the analysis of the equations for energy and linear momentum one can see that in the accompanying frame of reference these equations are reduced to the set of equations (6) and (7) for derivatives of entropy $s$. In this case equation (6) obtained from the energy equation defines the derivative of entropy along the trajectory, and equation 
(7), assigned to the equation for linear momentum, defines the derivatives of entropy in the direction normal to trajectory. As it will be shown below, derivatives of entropy obtained from the equations for energy and linear momentum have singularities. They turn out to be nonconjugated (i.e., they do not form a differential), and this points out to the nonconsistency of equations for energy and linear momentum. This fact follows from the anasysis of the relation obtained from equations (6) and (7), and this relation turns out to be nonidentical.

Such a nonidentical relation just enables one to study the consistency the conservation law equations and to discover the properties of solutions to the Euler and Navier-Stokes equations.

Equations (6) and (7) can be convoluted into the relation

$$
d s=\omega
$$

where $\omega=A_{\mu} d \xi^{\mu}$ is the first degree skew-symmetric differential form and $\mu=1, v$. Since the equations of conservation laws are evolutionary ones, the relation obtained is also an evolutionary relation. In this case the skew-symmetric form $\omega$ is also evolutionary one. [Skew-symmetric forms (such as $\omega$ ), which are obtained from differential equations, are defined on nonintegrable (accompanying) manifolds as opposed to exterior forms (Cartan, E., 1945). Such skew-symmetric forms, which are evolutionary ones, possess the properties that enable one to investigate differential equations. From those one can obtain closed inexact exterior forms, which are invariants and describe physical structures. This gives a possibility to understand the mechanism of origination of various physical structures (Petrova, L. I., 2008)].

\section{Nonidentity of the Evolutionary Relation. Inexact Solutions to Euler and Navier-Stokes Equations}

Evolutionary relation (10) is a nonidentical one as it involves the skew-symmetric differential form $\omega$, which is unclosed and cannot be a differential like the left-hand side of this relation. The evolutionary form $\omega$ is not closed since the differential of evolutionary form $\omega$ and its commutator are nonzero. The differential of evolutionary form $\omega$ is expressed as $d \omega=\sum K_{1 v} d \xi^{1} d \xi^{v}$, where $K_{1 v}$ are components of the form commutator. Without accounting for terms that are connected with the deformation of the manifold made up by the trajectories, the commutator can be written as

$$
K_{1 v}=\frac{\partial A_{v}}{\partial \xi^{1}}-\frac{\partial A_{1}}{\partial \xi^{v}}
$$

The coefficients $A_{\mu}$ of the form $\omega$ have been obtained either from the equation of the conservation law for energy or from that for linear momentum. This means that in the first case the coefficients depend on the energetic action and in the second case they depend on the force action. In actual processes energetic and force actions have different nature and appear to be inconsistent. The commutator of the form $\omega$ constructed of the derivatives of such coefficients is nonzero. (Below it will be shown what contribution into the commutator is made from the terms in coefficients $A_{\mu}$ and how these terms effect on description of the gas-dinamic system state). Since the commutator of form $\omega$ is nonzero, this means that the differential of the form $\omega$ is nonzero as well. Thus, the form $\omega$ proves to be unclosed and is not a differential. In the left-hand side of relation (10) it stands a differential, whereas in the right-hand side it stands an unclosed form that is not a differential. Such a relation cannot be an identical one.

As a result, even without a knowledge of particular expression for the form $\omega$ one can state that for actual processes the evolutionary relation turns out to be nonidentical. (Here it slould be emphasized that the equation obtained will be nonidentical regardless to with what accuracy the conservation law equations were written.)

Nonidentity of the evolutionary relation means that the equations of conservation laws for energy and linear momentum (entered into the set of Euler and Navier-Stokes equations) appear to be inconsistent. They cannot be convolved into identical relation for obtaing a differential. This points out to the fact that the Euler and NavierStokes equations turn out to be nonintegrable.

Below it will be shown that, when the additional conditions are realized, the Euler and Navier-Stokes equations can become locally-integrable ones.

Without additional conditions the solutions to Euler and Navier-Stokes equations cannot be a function (depending only on coordinates). That is, such solutions will be not exact ones. They will depend on the commutator of form $\omega$, which is nonzero. (Nonidentity of the evolutionary relation, and respectively nonintegrability of the Euler and Navier-Stokes equations, are due to the fact that the form $\omega$ is not closed, i.e. its commutator is nonzero.)

[It should be noted that in studing a cosistence of the equations entered into the set of Euler and Navier-Stokes equations necessary to consider the equation of mass conservation (the equation of continuity). In this case one 
obtains a relation that contains the second-degree skew-symmetric form in the right-hand side. In addition, it should be noted that in description of gas-dynamic system necessary to take into account the conservation law for angular momentum. In this case the right-hand side of evolutionary relation will include the third-degree skewsymmetric form. In the general case the evolutionary relation can be written as $d s=\omega^{p}$, where the form degree $p$ takes the values $p=1,2,3$.]

\section{Degenerate Transformations. Exact Solutions to the Euler and Navier-Stokes Equations}

The Euler and Navier-Stokes equations can have exact solutions only in the case when from the evolutionary skewsymmetic form $\omega$ in the right-hand side of nonidentical evolutionary relation it is realized a closed skew-symmetric form, which is a differential. In this case the identical relation is obtained from the nonidentical relation, and this will point out to a consistence of the conservation law equations and to an integrability of the Euler and NavierStokes equations.

Since the differential of evolutionary form (which is unclosed) is nonzero and the differential of closed exterior form equals zero, the closed exterior form can be obtained from evolutionary form only under degenerate transformation, namely, under a transformation that does not onserve differential. (The Legendre transformation is an example of such transformation.)

Degenerate transformations can take place under additional conditions, which relate to degrees of freedom. To this case a vanishing of such functional expressions as determinants, Jacobians, Poisson's brackets, residues and others can be assigned.

The conditions of degenerate transformation can be realized under change of nonidentical evolutionary relation, which appears to be a selfvarying relation. (Since one of the objects of evolutionary relation is an unmeasurable quantity, the other cannot be compared with the first one, and hence, the process of mutual variation cannot be terminated.)

The conditions of degenerate transformation specify the integral surfaces (pseudostructures): the characteristics (the determinant of coefficients at the normal derivatives vanishes), the singular points (Jacobian is equal to zero), the envelopes of characteristics of the Euler equations and so on.

The realization of the conditions of degenerate transformation means that it is realized a pseudostructure (a closed dual form), which describes an integral structure or surface. This occurs under degenerate transformation when the closed inexact (defined only on a pseudostructure) exterior form (with the differential being equal to zero) is obtained from the evolutionary form (with the differential being nonzero).

On the pseudostructure $\pi$, from evolutionary relation (10) it follows the identical relation

$$
d_{\pi} s=\omega_{\pi}
$$

Here the subscript $\pi$ points out to that the pseudostructure (integral surface) has been realized, and $\omega_{\pi}$ is a closed inexact exterior form. Since the form $\omega_{\pi}$ is a closed on pseudostructure form, this form turns out to be a differential. (It should be noted that such a differential is an interior one: it asserts only on pseudostructure, which is defined by the condition of degenerate transformation). There are differentials in the left-hand and right-hand side of this relation. This means that the relation obtained is an identical one.

Here the following is noteworthy. The degenerate transformation, under which a closed exterior form is obtained from evolutionary form, is realized as a transition from nonintegrable accompanying manifold (on which the evolutionary form is defined) to the integrable structures and surfaces with a closed form. Mathematically to this it is assigned a transition from one frame of reference to another nonequivalent frame of reference (from accompanying frame of reference to a locally-inertial on integral structures obtained).

Thus one can see that under degenerate transformation from nonidentical relation obtained from the conservation law equations for energy and linear momentum the identical relation is realized on pseudostructure (on integral structure). This means that the conservation law equations for energy and linear momentum become consistent, and this points out to realization of integrability of the Euler and Navier-Stokes equations.

In this case on the integral structure the solution to Euler and Navier-Stokes equations becomes exact one, i.e. it is a function (depending only on coordinates). This is so-called generalized solution. This solution is descrete one since it is realized only under additional conditions. As the relevant functions are defined only on integral structures or surfaces (made up by integral structures), these functions or their derivatives suffer a discontinuity 
along the directions being normal to integral structures or surfaces. It is evident that such discontinuites are described by the evolutionary form commutator.

Here it must be emphasized that inexact solutions to the Euler and Navier-Stokes equations are solutions with derivatives defined on tangent (nonintegrable) manifold whereas the derivatives of generalized functions are defined on integrable structures that can be only on cotangent (integrable) manifold. One can see that the transition from inexact solution to exact solution is a transition from tangent to cotangent manifold. (Here it is worth noting, that coordinates on integrable structures are not identical to the independent coordinates of the initial space on which equations defined.)

Thus, it has been shown that the Euler and Navier-Stokes equations have solutions of two types.

The further analysis of the evolutionary relation allows to disclose a physical meaning of such solutions and their role in evolutionary processes proceeding in gas-dynamic systems.

\section{Mechanism of Evolutionary Processes in Gas-dinamic System. Physical Meaning of Solutions to the Euler and Navier-Stokes Equations}

It has been shown that from the Euler and Navier-Stokes equations it follows the evolutionary relation, which solves the problem on integrability of the Euler and Navier-Stokes equations, i.e. on consistence of the equations entered into the sets of Euler and Navier-Stokes equations.

It appears that the evolutionary relation in addition solves another problem.

As the Euler and Navier-Stokes equations involve the conservation law equations, the problem on consistency of the Euler and Navier-Stokes equations is the problem on commutativity of conservation laws. Because of this, the evolutionary relation, which answers the question of consistency of the conservation law equations, describes in addition a commutativity of conservation laws. This discloses a role of conservation laws in evolutionary processes and a physical meaning of solutions to the Euler and Navier-Stokes equations.

\subsection{Nonequilibrium of Gas-dynamic System. Physical Meaning of the First Type Solutions to the Euler and Navier- Stokes Equations}

The evolutionary relation obtained from the conservation law equations possesses unique properties. This is due to the fact that this relation includes the entropy, which specifies the state of gas-dynamic system.

If evolutionary relation (10) be identical, from that one would obtain the differential of entropy $d s$ and find entropy $s$ as a function of space-time coordinates. It is precisely the entropy that will be the gas-dynamic function of state. The availability of the gas-dynamic function of state would point to the equilibrium state of gas-dynamic system. [Notice that the entropy, which depends on thermodynamic variables, enters into the relation (1) descriptive of the thermodynamic system state. And the entropy in the evolutionary relation for gas-dynamic system depends on space-time coordinates since the differential of entropy $d s$ in evolutionary relation has been made up by derivatives of entropy with respect to space-time coordinates. The entropy, which depends on thermodynamic variables, is a state function of thermodynamic system, and the entropy, which depends on space-time coordinates, is a state function of gas-dynamic system. In the gas dynamic system the entropy as a thermodynamic function specifies the state of gas (i.e. elements of gas-dynamic system) rather then of the gas-dynamic system.]

As has been shown above, the evolutionary relation is not identical. From such a relation the differential of entropy $d s$ cannot be defined. This points out to an absence of the gas-dynamic function of state and means that the gas-dynamic system state is nonequilibrium.

On the other hand, as this has been shown, the nonidentity of evolutionary relation points out to the fact that without additional conditions the solutions to Euler and Navier-Stokes equations are solutions of first type, i.e., they are inexact solutions.

As a result, the solutions of first type to Euler and Navier-Stokes equations, which are inexact, are assigned to non-equilibrium state of gas-dynamic system.

It was noted above that the nonidentical evolutionary relation is selfvarying one. Selfvarying of evolutionary relation points out to the fact that the gas-dynamic system state changes. However, since the nonidentity of evolutionary relation holds, the gas-dynamic system state remains nonequilibrium. (Such a change of non-equilibrium state of gas-dynamic system can lead to increasing nonequilibrium and developing instability.)

The nonequilibrium means that in gas-dynamic system an internal force acts. It is evident that the internal force 
is described by the commutator of skew-symmetric form $\omega$. (If the evolutionary form commutator be zero, the evolutionary relation would be identical, and this would point out to the equilibrium state, i.e. the absence of internal forces.) Everything that gives a contribution into the evolutionary form commutator leads to emergence of internal force that causes the non-equilibrium state and leads to development of instability.

[The nonidentity of evolutionary relation, which points out to non-equilibrium state of gas-dynamic system, discloses a role of conservation laws in such processes. Since the evolutionary relation has been obtained from the conservation law equations, the nonidentity of evolutionary relation points out to the fact that these equations are not consistent. And this means that the conservation laws under consideration are noncommutative ones. The reason of noncommutativy of conservation laws is due to the fact that the gas-dynamic system is subjected to actions of different nature, and in this case the nature of these actions are incompatible with the nature of the gas-dynamic system inself (i.e. these actions are not potential ones). Such actions cannot be directly converted into quantities of gas-dynamic system itself, and they cannot accumulated into that as a certain unmeasurable quantity which acts like internal force and leads to arising nonequilibrium.]

\subsection{Transition of the Gas-dynamic System into a Locally-equilibrium State}

As it has been shown above, under degenerate transformation the identical relation is obtained from nonidentical one.

From identical relation one can obtain the differential of entropy $d s$ and find entropy $s$ as a function of space-time coordinates. It is precisely the entropy that will be a gas-dynamic function of state. The availability of gas-dynamic function of state would point out to equilibrium state of gas-dynamic system. However, since the identical relation is satisfied only under additional conditions, such a state of gas-dynamic system will be a locally-equilibriud one.

One can see that the transition from nonidentical relation to identical one points out to transition of material system from non-equilibrium state into locally-equilibrium state.

On the other hand, as it has been shown above, the transition from nonidentical relation to identical points out to a transition from inexact solutions of the first type to exact solutions.

It turns out that the transition from inexact solutions to exact (generalized) silutions is assigned to the transition of gas-dynamic system from non-equilibrium state to locally-equilibrium state.

4.3 Mechanism of Arising Physical Structures and Appearance of Observable Formations. Physical Meaning of Generalized Solutions to Euler and Navier-Stokes Equations

Since the non-equilibrium state has been induced by an availability of internal force and in the case of locallyequilibrium state there is no internal force (in local domain of gas-dynamic system), it is evident that under transition of gas-dynamic system from non-equilibrium state into locally-equilibrium state the nonmeasurable quantity, which acted as internal force, changes to an measurable quantity. This manifests itself in the form of arising a certain observable, measurable, formation. Waves, vortices, turbulent pulsations and so on are examples of such formations.

[This conclution follows from the analysis of evolutionary relation. Closed dual forms and closed inexact exterior forms, which are realized under degerate transformations, made up a differential-geometric structure, i.e. a pseudostructure (integral structure) with conservative quantity (closed exterior form describes a consevative quantity because its differential equals zero). Realization of such differential-geometric structure (under degenerate transformation) points out to emergence of physical structure. The characteristics, the singular points, the envelopes of characteristics, and other structures with conserved quantities are examples of such physical structures. The origination of physical structure reveals as a new measurable and observable formation that spontaneously arises in gas-dynamic system. (It should be noted that physical structures and observed formations are not identical objects. Whereas the wave is an observable formation, to the physical structure the eikonal is assigned, that is, the moving wave element made up by physical structure).]

Exact generalized solutions to the Euler and Navier-Stokes equations describe such observable quantities arizen.

It is evident that the transition from inexact solutions to exact (generalized) solutions is assigned to a transition of gas-dynamic system from a non-equilibrium state to a locally-equilibrium state, which describes the process of emergence of observable formations, and the formation arisen (as it can be emphasized once more) are described by generalized solutions to the Euler and Navier-Stoles equations. In this case the discontinuities of function, which corresond to generalized solutions, or their derivatives are defined by a quantity, which is described by the 
commutator of unclosed form $\omega$ and acted as an internal force. Such a quantity defines the intensity of formations arisen (if the commutator be equal to zero, the intensity of formation would be equal to zero, i.e. the formation couldn't arise).

The process of arising observable formations discloses a mechanism of such phenomena as an emergence of vorticity and turbulence. (Below some concrete data for ideal and viscous gas will be presented.)

[Arising observable quantities is a change of inherent quantities of gas-dynamic system.

Assume that at a certain instant of time the local domain of gas-dynamic system is in equilibrium. That is, its quantities, for example, energy and linear momentum are consistent and simultaneously measurable ones. Then, under influence of some external (with respect to local domain) forces physical quantities changed and ceased to be consistent, measurable, quantities (inherent quantities of material system). When the degrees of freedom are realized, this allows physical quantities changed under external actions to redistribute in such a way that they become measurable physical quantities, i.e. inherent (corresponding to the nature of material system) quantities of material system. It is evident that the transition from initial measurable physical quantities to new physical quantities being realized is a discrete one. That is, inherent measurable physical quantities are changed discretely. These discrete changes of physical quantities manifest themselves as a certain formation arisen.]

Here the following should be noted. As it has been already pointed out, the transition from inexact solution to exact solution is a transition from tangent to cotangent manifold. From this follows that an emergence of observable formations can be described as transition from tangent manifold to cotangent one.

Mathematically, such a transition (i.e. the process of originating observable quantities) may be described by using two frames of reference, namely, an inertial system, in which the Euler and Navier-Stokes equations are written, and an accompanying frame of reference related to manifold made up by trajectories of elements (particles) of gas-dynamic system. Here it should be emphasized that both inexact solutions and exact generalized solutions are described in the inertial frame of reference. However, in doing so the coordinates of initial inertial frame of reference and the coordinates on integral structures are distinquished. The transition from the coordinates of inertial frame of reference (in which the Euler and Navier-Stokes equations and inexact solutions to these equations are written) to another coordinates of inertial frame of reference (in which the generalized solutions to the Euler and Navier-Stokes equations are received) turns out to be possible due to using the additional accompanying frame of reference.

Thus, the analysis of evolutionary relation obtained from the Euler and Navier-Stokes equations showed that these equations have solutions of two types, namely, inexact solutions being assigned to nonequilibriun state of gasdynamic system and exact generalized solutions, which corresponds to the locally-equilibrium state of gas-dynamic system. In this case the transition from inexact solution to exact one poits out to the transition of gas-dynamic system from non-equilibrium state to locally-equilibrium state and this process is accompanied by emergence of observable formations, which are described by generalized solutions.

Such properties of solutions to the Euler and Navier-Stokes equations enable one to disclose the mechanism of development of instability and arising vorticity and turbulence.

Below, by analysis of coefficients in the evolutionary relation for Euler and Navier-Stokes equations, it will be shown what evolutionary processes may proceed in the gas-dynamic systems of ideal and viscous gases.

\section{Gas-dynamic System of Ideal Gas}

It has been shown that a cause of the gas-dynamic nonequilibrium and instability is something that contributes into the commutator of the form $\omega=A_{\mu} d \xi^{\mu}$ contained in evolutionary relation obtained from the conservation laws for energy and linear momentum. In the case of ideal gas coefficients $A_{1}$ and $A_{v}$ are expressed by expessions (3) and $(8,9)$ respectively.

From the analysis of the expression $A_{v}$ and with taking into account the equality $A_{1}=0$, one can see that the terms related to the multiple connectedness of the flow domain (the second term in expression (8)), the nonpotentiality of the external forces (the third term in (8)) and the nonstationarity of the flow (the forth term in (8)) contribute to the commutator. All these factors lead to the emergence of internal forces, the non-equilibrium state and the development of various types of instability.

One can see that the development of instability is caused by the not simple connectedness of the flow domain, the nonpotential external (for each local domain of the gas-dynamic system) forces and the nonstationarity of the flow. 
(In the common case, the thermodynamic, chemical, oscillatory, rotational and translational nonequilibrium will effect on the gas-dynamic instability. It should be noted that in adddition it is necessary to consider the evolutionary relations that correspond to the conservation laws for angular momentum and mass as well.)

(It may be noted that, for the case of ideal gas, Lagrange (Kochin, N. E., Kibbel, I. A., \& Rose, N. V., 1963) derived the condition of the eddy-free stable flow. This condition is as follows: the domain must be simply connected one, forces must be potential and the flow must be stationary. One can see that under fulfillment of these conditions there are no terms that contribute into the commutator).

And yet, for every type of instability one can find an appropriate term giving contribution into the evolutionary form commutator, which is responsible for this type of instability. Thus, there is an unambiguous connection between the type of instability and the terms that contribute into the evolutionary form commutator in the evolutionary relation.

For example, at flow along the body by the flux of ideal gas the development of instability may be caused by the multiple connectedness of flow domain and the nonpotentiality of body forces. For $U>a$ ( $U$ is the velocity of the gas particle and $a$ is the speed of sound) the set of equations of the conservation law belongs to the hyperbolic type, and hence the transition to the locally-equilibrium state is possible on the characteristics and on the envelopes of characteristics as well, and weak and shock waves are formations of the system. If $U<a$, when the equations are of elliptic type, such a transition is possible only at singular points. The formations emerged due to the convection are of vortex type. At long acting the large-scale structures can be produced.

One can see that in gas-dynamic system, even in the case of ideal gas, it can originate the physical structures and relevant formations that lead to emergence of vorticity.

While studying the effects connected with the origination of the vorticity one can notice a certain specifics of numerical solving the Euler equations. In the case of ideal gas we have the equation for entropy, i.e. the energy equation $\partial s / \partial \xi^{1}=0$ (see, equation (6) and expression (3)). It seems that from this equation one can make a conclusion that, if initial conditions are the same in all trajectories, the entropy must be constant during all flow time. However, in actual cases (unsteady flow, flow along the body, heterogeneous medium) the derivative of entropy along the direction normal to trajectory suffers the break. Thus we have that, from one hand, entropy must be constant and, from other hand, its derivatives suffer breaks. This contradiction can be disclosed by taking into account the consistence of the Euler equations (Petrova, L. I., 2009).

[Here one should keep in mind that entropy, which obeys to a single equation, namely, the conservation law for energy, is not a function (it depends on trajectory rather then only on the space-time coordinates). Entropy will be a function (and state function) only if it will simulatneously obey two equations, that is, in addition it will obey to the conservation law equation for momentum.]

[Studying the instability on the basis of analysis of entropy behavior was carried out in the works by I.Prigogine and co-authors (Prigogine, I., 1955; Glansdorff, P. \& Prigogine, I., 1971). In paper (Prigogine, I., 1955) (for which the Nobel prize has been awarded to I. Prigogine) it has been shown that the quantity (named excessive entropy), which is described by the commutator made up of mixed derivatives of entropy with respect to the thermodynamic variables) leads to development of thermodynamic instability. That is, in that paper the entropy, which depends on thermodynamic variables, (i.e. the thermodynamic entropy) was considered and the thermodynamic instability was studied. And in paper (Glansdorff, P. \& Prigogine, I., 1971) I. Prigogine and co-authors tried to investigate the development of instability in gas-dynamic systems with the help of thermodynamic entropy (i.e. entropy depending on thermodynamic variables). The entropy was considered as a thermodynamic function of state (though its behavior along the trajectory was analyzed). By means of such state function one can trace the development (in gas fluxes) of the thermodynamic instability only. To investigate the gas-dynamic instability it is necessary to consider the entropy depending on space-time coordinates. Whereas for studying the thermodynamic instability one has to analyze the commutator made up of mixed derivatives of entropy with respect to thermodynamic variables, for studying the gas-dynamic instability it is necessary to analyze the commutators made up of mixed derivatives of entropy with respect to the space-time coordinates.]

\section{Gas-dynamic System of Viscous Gas}

In the case of ideal gas the expression $A_{1}$ in the equation of energy (see equations (6) and formulae (3)) is equal to zero. In the case of a viscous heat-conductive gas the expression $A_{1}$, as one can see from expression (4) obtained using the Navier-Stokes equations, depends on viscosity and heat-conductivity. In the case of viscous gas the 
terms related to transport phenomena (viscous and heat-conductive) will contribute into the evolutionary form commutator. These terms are responsible for emergence of turbulent pulsations.

In the general case, the expression $A_{1}$ will include the terms accounting for the chemical, oscillatory, rotational, translational, and other effects (Clark, J. F. \& Machesney, M., 1964) that will contribute into the evolutionary form commutator and influence on the development of instability.

Let as analyze the following example.

Boundary layer. The instability originates due to the multiple connectness of the domain and the transport phenomena (the effect of viscosity and thermal conductivity). Contributions into the commutator produce the second and third terms in expression (4) and the terms related to the multiple connectedness of the flow domain (the second term in expression (8)). The transition to the locally equilibrium state is allowed at singular points because in this case $\partial s / \partial \xi^{1}=A_{1} \neq 0$, that is, the external exposure acts onto the gas particle separately, the development of instability and the transitions to the locally-equilibrium state are allowed only in the individual fluid particle. Hence, the formations emerged behave as pulsations. These are turbulent pulsations.

[It is commonly believed that the instability is an emergence of any structures in the gas-dynamic flow. From this viewpoint the laminar boundary layer is regarded as stable one, whereas the turbulent layer can be regarded as unstable layer. However the laminar boundary layer cannot be regarded as a stable one because of the fact that due to the not simple connectedness of the flow domain and the transport processes the instability already develops although any formations do not originate. In the turbulent boundary layer the emergence of pulsations is a transition to the locally-equilibrium state, and the pulsations themselves are local formations. The other matter, due to the global nonequilibrium the locally-equilibrium state is broken up and the pulsations weaken.]

Commonly, attempts to describe the rise of turbulence using the Navier-Stokes equations involved difficulties and leaded to idea that for solving this problem additional equations are required. However, this approach also didn't give reasonable results.

From the present paper it follows that the Navier-Stokes equations describe a mechanism of turbulence origination. However, in this case there is a subtle point. It has been shown that an origin of any observable formations, including turbulent pulsations, turns out to be related to transition from tangent manifold of differential equations to cotangent one. Such a transition is related to the degenerate transformation and cannot be described numerically by continuous procedure. Actually, at numerical solving the simulation of derivatives of differential equations by the difference methods proceeds on tangent manifold. In this case one can obtain only inexact solutions to the Navier-Stokes equations. And, to obtain turbulent pulsations and describe turbulence, one has to obtain exact solutions with derivatives defined on cotangent manifold (on which integral structures are defined). To do this, one has to take into account the consistence of equations entered into the set of Navier-Stokes equations.

\section{Conclusion}

Thus, it has been found that the Euler and Navier-Stokes equations have solutions of two types, namely, inexact solutions being assigned to nonequilibriun state of gas-dynamic system and exact generalized solutions, which corresponds to the locally-equilibrium state of gas-dynamic system. In this case the transition from inexact solution to exact one points out to the transition of gas-dynamic system from non-equilibrium state to locally-equilibrium state and this process is accompanied by emergence of observable formations, which are described by generalized solutions. This discloses the mechanism of origin of such processes as vorticity and turbulence.

In conclusion it should be said a little about numerical sumulation of unstable flows.

As it was already mentioned, in numerical solving the differential equations the modelling with difference method proceeds on tangent manidold, whereas the formations arised are realized on integral structures being related to cotangent manifold. For this reason, in numerical solving it appears to be impossible to obtain the structures and formations arisen by a continuous way.

In numerical simulation of the gas flow care should be taken to the condition under which the consistence of equatuins is satisfied. The realization of this condition will point out to origination of any formations and allow to obtain the solution describing these formations.

It is possible to describe the emergence of vorticity and turbulence when using simultaneously two methods: the method of direct numerical simulating the Euler and Navier-Stokes equations (Paskonov, V. M., Petrova, L. I., \& Polyansky, B. A., 1985), which is fulfilled on tangent manifold and enables one to obtain inexact solutions and the 
method of solving the Euler and Navier-Stokes equations when the equations are provided with the integrability conditions (the conditions of consistence) and this enables one to obtain integral structures or surfaces, that is, to go out onto cotangent integrable manifold and obtain exact solutions (the methods of characteristics, symmetries, eigen-functions and others are examples of such methods).

This gives a possibility to obtain the transitions from inexact solutions to exact ones, and thus to describe the process of origination of vorticity and turbulemce (i.e. the process of emergence of any observable formations).

\section{References}

Cartan, E. (1945). Les Systemes Differentials Exterieus ef Leurs Application Geometriques. Paris, Hermann.

Clark, J. F., \& Machesney, M. (1964). The Dynamics of Real Gases. London: Butterworths.

Glansdorff, P., \& Prigogine, I. (1971). Thermodynamic Theory of Structure, Stability and Fluctuations. New York: Wiley.

Haywood, R. W. (1980). Equilibrium Thermodynamics. Wiley Inc.

Kochin, N. E., Kibbel, I. A., \& Rose, N. V. (1963). Theoretical Hydrodynamics. Part 1, FM.

Liepman, H. W., \& Roshko, A. (1957). Elements of Gas Dynamics. New York: Jonn Wiley.

Paskonov, V. M., Petrova, L. I., \& Polyansky, B. A. (1985). Application of the Navier-Stokes model in numerical simulation of a viscid gas flow with account for chemical processes. Moscow State University.

Petrova, L. I. (2008). The mechanism of generation of physical structures. Nonlinear Acoustics-Fundamentals and Applications (18th International Symposium on Nonlinear Acoustics, Stockholm, Sweden, 2008). New York, American Institute of Physics (AIP), pp. 151-154.

Petrova, L. I. (2009). Relationships between discontinuities of derivatives on characteristics and trajectories. J. Computational Mathematics and Modeling, 20(4), 367-372. http://dx.doi.org/10.1007/s10598-009-9043-0

Prigogine, I. (1955). Introduction to Thermodynamics of Irreversible Processes. C. Thomas, Springfild. 